\title{
THE FAN PROPELLER AND BLADE INTERFERENCE.
}

\author{
BY M. A. S. RIACH, F.R.Ä̈.S., ASSOC.INST.N.A.
}

For some time it has been recognised that the aerodynamical theory of the screw propeller, as at present presented, leaves considerable room for improvement. The leading specialists in this branch of aerodynamics, such as Mr. Fage, Mr. McKinnon-Wood and Dr. Watts, have given recognition to the all-important fact that a velocity of inflow can only be produced by the mutual interfering action of the propeller blades upon each other. Dr. H. C. Watts was, I believe, the first person to point this out clearly, and to show that the hitherto accepted theories of inflow rested upon a highly empirical basis. By "velocity of inflow" is meant the additional velocity required to modify the method of propeller analysis, known as the blade element theory, originally enunciated by $S$. Drzewiecki in 1892 . The accepted and standard inflow theory of the airscrew, as, for example, given in Professor L. Bairstow's "Applied Aerodynamics," rests upon the empirical assumption that the ratio (velocity of inflow)/(velocity of impressed slip) is a constant for all propellers and for all radii along the propeller blade length. The numerical value of this ratio it has been left to experimental research to determine, but fundamentally it is nothing more nor less than an empirical factor of ignorance to be justified only by the sparcity of time and the pressure of work during the war period. It is time that a more rational, because more fundamental, theory was evolved. In attempting to produce such a theory I have started out with two basic postulates:-

(I) The blade element theory of Drzewiecki is correct, to a sufficient degree of approximation, if blade interference be supposed to be nonexistent.

(2) The physical action of an airscrew may be represented, to a good degree of approximate accuracy, by incorporating with (I) the effects of blade interference.

I consider here only the special case of a propeller working without axial advance in a fluid, i.e., what has been called the "static" case. The general case of propeller action is of necessity more difficult, and it is thought that the present paper will have achieved its object if it suggests a line of inquiry which may prove fruitful in the general case as judged by the results given by the theory in the static case of screw action.

It is well known that if any symmetrical body be moving uniformly through air at rest, some air will be pushed along in front of the body and continue to be pushed along so long as the action continues. Conversely, if air be moving past a body at rest, some of the air in the neighbourhood of the body will be slowed up and this will continue so long as the action lasts. In both cases the action upon the air, in the neighbourhood of the body, gives rise to a force which is known as the resistance of the body to the motion. If $U$ be the velocity of the body relatively to the air a long way in front of it, and $\Sigma$ be the velocity which the body impresses upon the air, then the change in energy per second in the relative air stream is $\frac{1}{2}$ (mass of air per second affected) $\left[U^{2}-(U-\Sigma)^{2}\right]$, the resistance force is (mass of air per second affected) $\Sigma$, and the work done per second by the resistance force upon the air is (resistance) $V$, where $V$ is the relative velocity. between the body and the air at the body. Then if the work done by the body 
upon the air is equal to the change in energy in the relative air stream, we have simply :-

$$
\Sigma V=\frac{1}{2}\left[U^{2}-(U-\Sigma)^{2}\right]
$$

i.e., $\quad V=U-\Sigma / 2$, which gives the relative velocity at the body. Now the above is a very elementary conception and in practice the conditions are far more highly complex. It is intended by way of illustration only.

Consider now a more difficult case, the case of an aerofoil moving uniformly through air at rest or, conversely, air moving uniformly past an aerofoil at rest in it. The same general type of thing occurs here (Fig. I).

I call $\gamma$ the gliding angle of the aerofoil, so that $: \tan \gamma=\mathrm{drag} / \mathrm{lift}$. Then it is seen that the component velocities, normal and tangential to the direction of the resultant force upon the aerofoil, are $U \cos \gamma$ and $U \sin \gamma$. We may, by way of illustration, consider the component velocity $U \sin \gamma$ to be analogous to the free wind velocity of the case already considered and then we may expect this velocity to be slowed up as it passes the aerofoil, the other component velocity $U \cos \gamma$ remaining unchanged in value.

The aerofoil, as it moves, pushes some air along with it, but principally downwards. Call this impressed velocity $\Sigma$, and let part of it be present at the aerofoil and call this part $\sigma \Sigma$. Then, if we pursued the same reasoning as before, we should find the value of $\sigma$ to be one-half. At present we shall leave it indeterminate in value. Then the relative component velocity at the aerofoil is not $U \sin \gamma$, but $[U \sin \gamma-\sigma \Sigma]$ (Fig. 2).

Hence the resultant velocity at the aerofoil is now seen to be inclined to the free wind direction at an angle of $\xi$ and to be equal to

$$
W=\sqrt{ } U^{2} \cos ^{2} \gamma+(U \sin \gamma-\sigma \Sigma)^{2}
$$

Notice that this resultant velocity $W$ at the aerofoil may be constructed either by the velocities $U \cos \gamma$ and $(U \sin \gamma-\sigma \Sigma)$, or by the velocities $U$ and $\sigma \Sigma$. The component velocity $U \sin \gamma$ is slowed up as it passes the aerofoil by the amount of the impressed velocity $\sigma \Sigma$ so that the component relative velocity at the aerofoil is $(U \sin \gamma-\sigma \Sigma)$.

Call the angle of chord incidence to the free wind direction $a_{\mathrm{r}}$. Now I want to consider a hypothetical case in which it is to be supposed that a quantity of uniformly deflected air moves past the aerofoil in relative motion and with the relative velocity $W$. Let the depth of this layer of air be $\Delta$, and let the chord width of the aerofoil element over which this air is supposed to flow in a twodimensional motion be $b$. Call $b / \Delta=\delta$. Then I propose to show that this ratio $\delta$ may be regarded tentatively as a general constant.* Consider an element of aerofoil surface of span $d x$. Then the volume of air flowing over this element per second will be $\Delta d x W$, its mass per second will be $\rho \Delta d x W$, and the resultant force upon the aerofoil element will then be $\rho \Delta d x W \Sigma$. Now write down the aerodynamical equation for the resultant force. It is

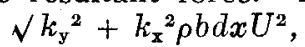

where $k_{\mathrm{y}}$ and $k_{\mathrm{x}}$ are the coefficients of lift and drag respectively and $\rho$ is the mass/density of the air. Then

which defines $\delta$. Also

$$
\begin{gathered}
\rho \Delta d x W \Sigma=\sqrt{ }\left({k_{\mathrm{y}}}^{2}+k_{\mathrm{x}}{ }^{2}\right) \rho b d x U^{2} \\
\text { i.e., } W \Sigma=\sqrt{ }\left({k_{\mathrm{y}}}^{2}+k_{\mathrm{x}}{ }^{2}\right) \delta U^{2},
\end{gathered}
$$

Hence

$$
\tan \xi=(\sigma \Sigma \cos \gamma) /(U-\sigma \Sigma \sin \gamma) \text { (see Fig. 2). }
$$

and

$$
\sigma \Sigma \cos \gamma=(U \tan \xi) /(\mathbf{I}+\tan \gamma \tan \xi),
$$

$$
\begin{gathered}
\therefore(W / \sigma)(U \tan \xi) /(\mathbf{I}+\tan \gamma \tan \xi)=k_{\mathrm{y}} \delta U^{2}, \\
\because \sqrt{ }\left(k_{\mathrm{y}}{ }^{2}+k_{\mathrm{x}}{ }^{2}\right)=k_{\mathrm{y}} \sec \gamma .
\end{gathered}
$$

* It will be seen later that this is not strictly necessary. 


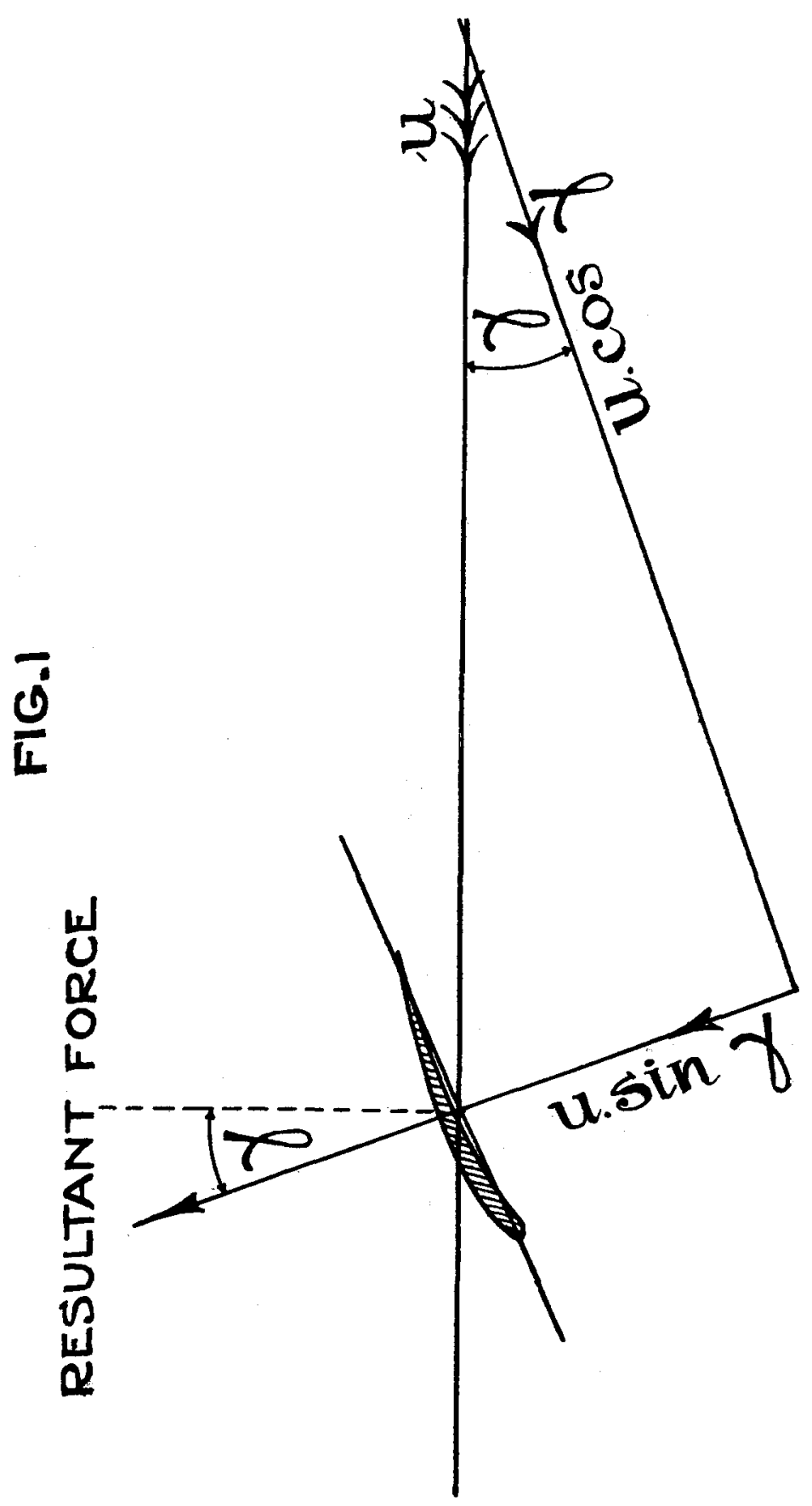







Further,

And

$$
W=U / \cos \xi(\mathrm{I}+\tan \gamma \tan \xi) \text { (see Fig. } 2) \text {. }
$$

$$
\begin{gathered}
\therefore(\mathrm{r} / \sigma) /\left(U^{2} \tan \xi \sec \xi\right) /(\mathrm{I}+\tan \gamma \tan \xi)^{2}=k_{\mathrm{y}} \delta U^{2} \\
i . e .,(\tan \xi \sec \xi) /(\mathrm{r}+\tan \gamma \tan \xi)^{2}=\sigma k_{\mathrm{y}} \delta
\end{gathered}
$$

or alternatively since $\tan \gamma=k_{\mathrm{x}} / k_{\mathrm{y}}$;

$$
\left(\tan \xi \sec \xi k_{\mathrm{y}}\right) /\left(k_{\mathrm{y}}+k_{\mathrm{x}} \tan \xi\right)^{2}=\sigma \delta .
$$

Now for the angles with which we shall have to deal, $\xi$ is always a small angle so that we can write approximately:-

$$
(\pi / \mathrm{I} 80) \xi k_{\mathrm{y}} /\left[k_{\mathrm{y}}+k_{\mathrm{x}}(\pi / \mathrm{I} 80) \xi\right]^{2}=\sigma \delta
$$

Solving the quadratic for $\xi$ we get to the same degree of approximation :-

$$
\xi=k_{y} \delta \sigma(\mathrm{r} 8 \mathrm{o} / \pi) /\left(\mathrm{r}-2 k_{\mathrm{x}} \sigma \delta\right)
$$

Now it is known, from experiments on "downwash" angles, that the angle of downwash varies as the lift coefficient almost exactly, i.e., that $\xi=\Omega k_{\mathrm{y}}$, where $\Omega$ is a constant for the aerofoil shape. Hence, $\Omega=(\mathrm{r} 80 / \pi) \delta \sigma /\left(\mathbf{r}-2 \delta \sigma k_{\mathrm{x}}\right)$, and $\left(\sigma \delta k_{x}\right)$ is small compared to unity, so that we may conclude that $\sigma \delta$ is a constant $\dagger$ and, if $\sigma$ be a general constant, then $\delta$ is a constant and tentatively may be assumed to be approximately a general constant.

This point, as already mentioned, I wished to demonstrate. The value of $\Omega$ has been found by experiment to be about 20 in round numbers, so that we can conclude tentatively that a value of $\sigma \delta=\pi / 9$ is of the right order. Note in passing that, $\delta$ being a ratio and the quotient of two linear quantities, its value can change only through change of the angle of incidence if it changes at allchange of chord width for example cannot affect its value because the type of flow round two geometrically similar aerofoils of different size is the same for equal incidence angles, except for small [ $[v l]$ effects which can présumably be ignored at the high speeds encountered in propeller action.

Hence, by substitution, we get to the same degree of approximation the value of $\Sigma$ given by

$$
\Sigma=\left\{U \delta \sqrt{ }\left(k_{\mathrm{y}}{ }^{2}+k_{\mathrm{x}}{ }^{2}\right)\right\} /\left(\mathrm{I}-\sigma \delta k_{\mathrm{x}}\right) ;
$$

so that the total impressed velocity $\Sigma$ is seen to vary as the free wind velocity and as the lift coefficient for most angles of incidence approximately.

\section{Application to Propeller Theory.}

To successfully study the mutual interfering action of the propeller blades upon each other it is necessary to consider them as forming an infinite series of equal members equally spaced apart and to consider the conditions at any member of the series which is far removed from the first member. We study here the cascade series corresponding to any one element of a blade of course. We make the assumption that the conditions at the $r$ th member of the series become "steady" as $r$ is increased indefinitely, and by this definition of " steady" we mean that the conditions at and after the $r$ th member are all identical. We do not, however, imply that the air flow is uniform round the circumference of any annulus in the screw disc, it is probably different at the blades to anywhere else in the annulus, but we assume that it is the same at any one blade as at any other blade (at equal radii of course). Such hon-uniformity in the airflow round any annular circumference gives rise to the familiar " gustiness" associated with all propeller action. Consider Fig. 3, which represents a cascade series of equal and equally spaced aerofoil elements.

By our definition of a "steady" state and by our assumption that such a state exists at and after the $r$ th member of the above series, we. know that the inflow velocities at all the members of the series, at and after the $r$ th member,

\footnotetext{
+ This is the important deduction.
} 
are identical. These inflow velocities are produced by the action of the $(r-1)$. members of the series, from and including the $(r-\mathrm{I})$ th member up to the first member of the series. They are the "legacies" of the impressed velocities produced by these $(r-1)$ aerofoils. Now since $r$ is a very large number, it is not to be expected that the action of the first member of the series will have any appreciable effect upon the $r$ th member. If we consider the aerofoils above to be moving with uniform speed $C$, from left to right through air at rest, it is evident that by the time the $r$ th member has reached the position $a a^{\prime}$ the impressed velocity produced by the first member will have practically died out and so have no appreciable action (in producing a speed of inflow) upon the $r$ th member. The same thing applies to the second, third, fourth, etc., members of the series. But when we get close enough to the $r$ th member the " neighbouring" members do have an appreciable effect upon the $r$ th member in producing a speed of inflow, i.e., the $(r-\mathrm{I})$ th, $(r-2)$ th, etc., members have such an appreciable effect. So that what we have virtually to try and do is to $8 u m$ a series having an infinite number of continuously decreasing terms. How are we to effect this without knowing the series to be summed? I suggest the following process as being extremely simple and having a rational basis. To begin with, the sum to infinity of the series is finite, for if not we should have, an infinite speed of inflow which is absurd. Hence, we may define a number $q$ (not necessarily integral) which shall represent the "mean" value corresponding to the sum to infinity. The definition of $q$ is :-

That is, if

$$
q=\frac{\text { speed of inflow at } r \text { th member }}{\sigma \text { (impressed velocity of } r \text { th member) }}
$$

$\Sigma_{\mathrm{r}}=$ impressed velocity of $r$ th member,

and

and if

$$
\sigma \Sigma_{r}=\text { the proportion of this velocity at the } r \text { th member, }
$$

$\Sigma_{\mathrm{s}}=$ speed of inflow at $r$ th member,

then $q$ is defined by

$$
q=\Sigma_{\mathrm{s}} / \sigma \Sigma_{\mathrm{r}}, \text { i.e., } \Sigma_{\mathrm{s}} / \Sigma_{\mathrm{r}}=\sigma q=\text { inflow/slip ratio. }
$$

In other words, what number multiplied by the impressed velocity at the $r$ th member will represent the sum to infinity of all of the impressed velocities at all the members from the $(r-1)$ th up to the first inclusive? The answer is $q$, which is thus defined-

Consider the $(r+q)$ th term.

Since the times taken for the $(r+q)$ th term to move into the positions initially occupied by the $(r+q-\mathbf{r})$ th, $(r+q-2)$ th, . . rth terms are small, provided $q$ is small compared to $r$, the velocities impressed by the $r$ th, $(r+I)$ th, . . $(r+q-1)$ th terms will not be sensibly different in value to the impressed velocities at these terms by the time the $(r+q)$ th term has moved over all the positions initially occupied by these terms. This is only another way of looking at the definition of $q$. Now obviously the value to be assigned to $q$ is not necessarily integral and will in general depend upon the value of the ratio $2 \pi x / N b$, where $x$ is the blade radius, $N$ the number of blades, $2 \pi x / N$ the "spacing" or distance apart of the members of the cascade series, and $b$ the chord width of each member. I now introduce a convention regarding $q$, for the sake of simplicity only and as a first tentative estimate as to its probable value which may later be discarded if required without interfering with the general theory developed here. $q$ is defined as the number of equivalent members of the series which may be looked upon as producing the inflow speed at the final member next to them (to the left, Fig. 3). Hence, consider Fig. 4. 





Suppose that we crowd in some more members between the $r$ th and the $\{r+q-1)$ th members, so that the new arrangement of all the members (new and old) is still an equally spaced arrangement and all the members are identical. Then, by my convention, I suppose that the effective number of members which now affect the $(r+q)$ th member is simply equal to the total of the members from $r$ to $(r+q-\mathrm{I})$, i.e., the sum of the old and new members. And similarly, if we thin out the original arrangement and decrease the total number of members from $r$ to $(r+q-\mathrm{I})$. Then the total distance from $r$ to $(r+q-\mathrm{I})$ being $2 \pi x q / N=2 \pi x b q / N b$, I suppose by this convention that $2 \pi x q / N$ is always of the same value so long as the value of $b$ remains the same. Now evidently if we increase $b$ we get the same effect as by crowding in more members, for then the "spacing" chord width ratio becomes smaller in the same way as by increasing the number of members. Hence, if we are to include the chord width $b$ in our convention for $q$ we must make $2 \pi x q / N b=$ constant. This is what we do and this then constitutes the convention regarding the value of $q$. Hence,

$$
q=N b \text { (constant) } / 2 \pi x=k(N b / x) .
$$

Hence now :-

Ratio (inflow speed)/(impressed slip speed) $=\sigma q=\sigma k(\mathrm{Nb} / \mathrm{x})$.

Then for most general designs of propeller blade shapes this ratio decreases as the radius increases and vice-versa, becoming infinite in value at the propeller boss. The type of curve of variation is very similar to that found experimentally by McKinnon Wood at the R.A.E. in his cascade experiments. The theory is seen to be in good agreement with experiment.

Now consider the conditions at the $(r+q)$ th aerofoil (Fig. 5) where the flow is " steady."

Let the angle of the aerofoil element to the horizontal (i,e., the plane of rotation of the propeller) be a. Then the inflow velocity $\Sigma_{8}$ makes the real angle of incidence of the aerofoil $a_{r}$. $C$ is the velocity of the element due to its rotation (the circumferential velocity) and is the same as the velocity $C$ of Fig. $3 . U$ is the free wind velocity of the aerofoil. $W$ is the relative velocity at the aerofoil. $\gamma$ is the gliding angle of the aerofoil at an incidence of $a_{r}$. The velocities $\sigma \Sigma_{r}$ and $\Sigma_{\mathrm{s}}$ lie at the same angle because the motion is "steady," and $\Sigma_{\mathrm{s}}$ is the sum of a number of equal $\sigma \Sigma_{\mathrm{r}}$ 's.

We can now find the value of $a_{r}$, the real angle of incidence of the aerofoil element. From the geometry of Fig. 5 we have:-

$$
\operatorname{Sin}\left(a-a_{\mathbf{r}}\right)=\left\{\Sigma_{\mathrm{s}} \cos \left(\gamma+a-a_{\mathbf{r}}\right)\right\} / U
$$

But $\Sigma_{g}=\sigma q \Sigma_{r}$, and $\Sigma_{r}$ is the same as the $\Sigma$ of the simple aerofoil considered at the beginning of this paper. Hence :-

and

$$
\Sigma_{\mathrm{r}}=\left\{U \delta \sqrt{ }\left(k_{\mathrm{y}}^{2}+k_{\mathrm{x}}^{2}\right)\right\} /\left(\mathrm{I}-\sigma \delta k_{\mathrm{x}}\right)=\left(U \delta k_{\mathrm{y}} \sec \gamma\right) /\left(\mathrm{I}-\sigma \delta k_{\mathrm{x}}\right) .
$$

$$
\begin{aligned}
& \therefore \sin \left(a-a_{r}\right)=\left\{\sigma q U \delta k_{y} \sec \gamma \cos \left(\gamma+a-a_{r}\right)\right\} /\left(\mathrm{r}-\sigma \delta k_{x}\right) U \\
& \text { i.e., } \tan \left(a-a_{\mathrm{r}}\right)=\left(\sigma q \delta k_{y}\right)\left[\mathrm{r}-\tan \gamma \tan \left(\alpha-a_{\mathrm{r}}\right)\right] /\left(\mathrm{r}-\sigma \delta k_{\mathrm{x}}\right)
\end{aligned}
$$

and

$$
\therefore \tan \left(\alpha-\alpha_{\mathrm{r}}\right)=\left(\sigma q \delta k_{\mathrm{y}}\right) /\left\{\mathrm{I}-\sigma \delta k_{\mathrm{x}}(\mathrm{r}-q)\right\}
$$

But to the same degree of approximation previously employed this becomes:-

$$
(\pi / \mathbf{1} 80)\left(a-a_{\mathbf{r}}\right)=\left(\sigma q \delta k_{\mathrm{y}}\right) /\left\{\mathrm{I}-\sigma \delta k_{\mathbf{x}}(\mathbf{I}-q)\right\}
$$

which gives the value of $a_{\mathrm{r}}$ in terms of $q$, since $k_{\mathrm{y}}=f\left(a_{\mathrm{r}}\right)$.

Now we can further simplify this result by assuming an equation for $k_{\mathrm{y}}$ in terms of $a_{r}$. For many types of aerofoils it is found that the lift coefficient is nearly a straight line from the angle of no-lift up to the stalling angle, so that let us put $k_{\mathrm{r}}=m\left(a_{\mathbf{r}}-\beta\right)$, where $m$ is a constant for the aerofoil defined by 


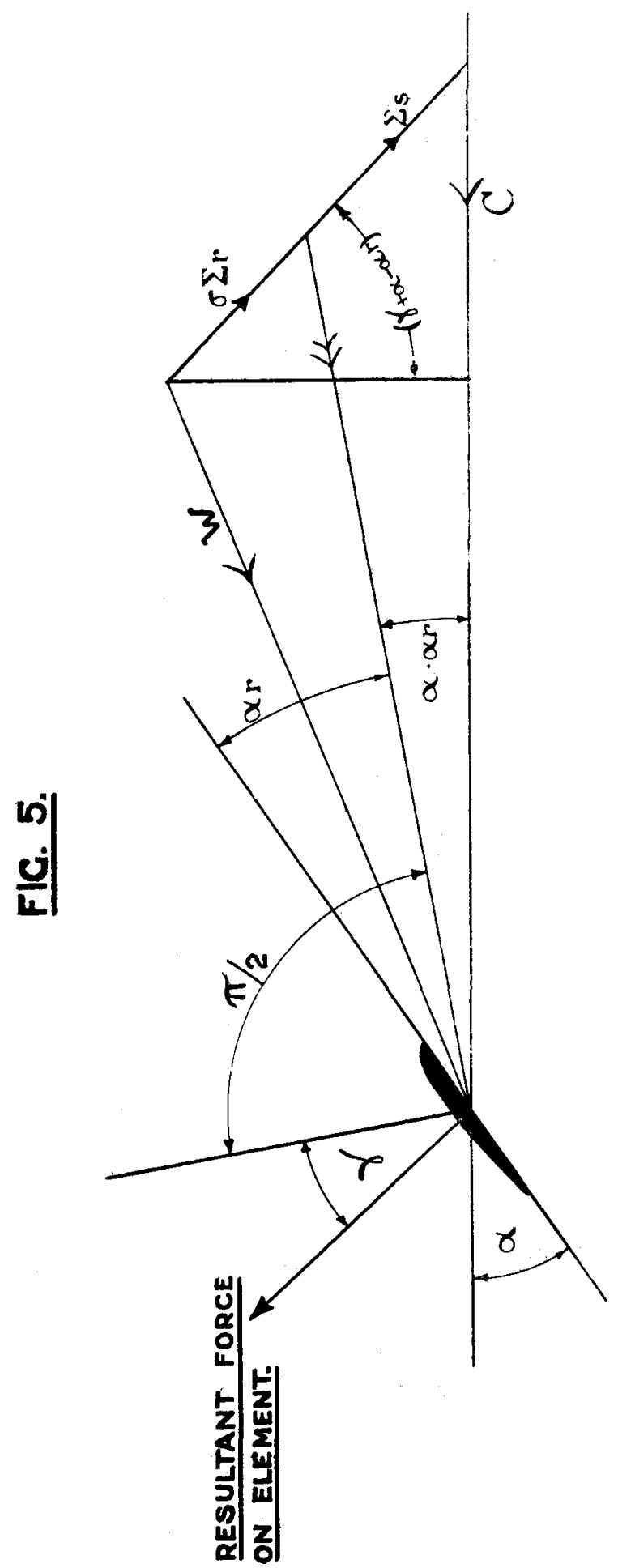


$\delta k_{\mathrm{y}} / \delta a_{\mathrm{r}}=m$, and $\beta$ is the angle at which $k_{\mathrm{y}}$ vanishes, i.e., the angle of no-lift of the aerofoil.

Then

$$
(\pi / 180)\left(a-a_{r}\right)=\left\{\sigma q \delta m\left(a_{r}-\beta\right)\right\} /\left\{1-\sigma \delta k_{x}(1-q)\right\}
$$

Now $k_{x}$ appears in the denominator and is associated with a term having a very small value compared to unity, unless $q$ is very large in value. Hence, we can tentatively neglect this term and write sufficiently approximately:-

giving

$$
(\pi / \mathbf{r} 80)\left(a-a_{\mathbf{r}}\right)=\sigma q \delta m\left(a_{\mathbf{r}}-\beta\right)
$$

where

$$
a_{\mathbf{r}}=(\alpha+\lambda q \beta) /(\mathbf{I}+\lambda q)
$$

$$
\lambda=(\mathrm{I} 8 \mathrm{o} / \pi) \sigma \delta m \text {. }
$$

This is the general solution for $a_{\mathbf{r}}$ for the static case of propeller action.

Now before we can find the numerical value of $a_{r}$ in any case we require to know the value of $q \lambda$, i.e., the value of $q \sigma \delta$, since the value of $m$ is known from the aerofoil shape. This unknown product can be settled experimentally if we make the substitution for $q:-$

$$
q=k(N b / x) \text {, so that } q \sigma \delta=\sigma \delta k(N b / x) .
$$

And we can find the value of $\alpha \delta k$ from our experiment. Then, knowing the value of $\sigma \delta k$, we know the value of $q \sigma \delta$ in any case and hence know the value of $a_{\mathrm{r}}$ in any case. Note that $q, \sigma$ and $\delta$ do not change in value with change of the angle of incidence.*

Consider now for a moment the derivation of $q$. The inflow velocity at any member of the series after the $(r+q)$ th member is constant and is the sum of a certain (variable) number $(q)$ of the impressed velocities at the members immediately in front (to the right) of it (Fig. 4). Thus, the inflow velocity at the $(r+q)$ th member is the sum of the impressed velocities at the $(r+q-1)$ th, $(r+q-2)$ th, . . r th members. And the inflow velocity at the $(r+q+1)$ th member is the sum of the impressed velocities at the $(r+q)$ th, $(r+q-\mathbf{I})$ th, ... $(\mathrm{r}+\mathrm{I})$ th members. Hence the inflow speed at any member after the $(r+q)$ th member is simply the sum of the impressed velocities at the $q$ members. immediately in front of it, and the point to notice is that this does not in general involve in this sum the impressed velocity at any one member such as the $r$ th member. If we take a member sufficiently far down (to the left of) the series after the $(r+q)$ th member, the impressed velocities at the $r$ th, $(r+r)$ th, etc., members do not contribute to the inflow speed at the member chosen because they are too far away to affect it. So that if we now ask the question, what is the speed of indraught (total flow speed) at any point on the circumference of any annulus on the disc circle, we cannot say it is the same thing as the indraught at any blade element because the indraught here is the sum of the inflow speed and the impressed speed at the blade element, which impressed speed forms a part of the inflow speed at the next blade element round the annulus. So that it seems probable that the indraught speed into any annulus is a maximum at the blade elements and a minimum between the blades. However, in view of the constancy of the inflow speed and the remarks made above, it seems reasonable to suppose that the mean indraught into any annulus approximates to the inflow speed and that the indraught speed at the blade elements is very local and falls off rapidly to the inflow speed value even close to the blade elements, which value it maintains over the annular circumference until the next blade element is reached when it again rises in value to the sum (inflow speed + impressed speed). Fig. 6 illustrates what is meant. We shall then tentatively assume the mean speed of indraught into any annulus on the disc circle to be approximately equal to the

* Strictly this should read " $q$ and $\sigma \delta$, etc." 


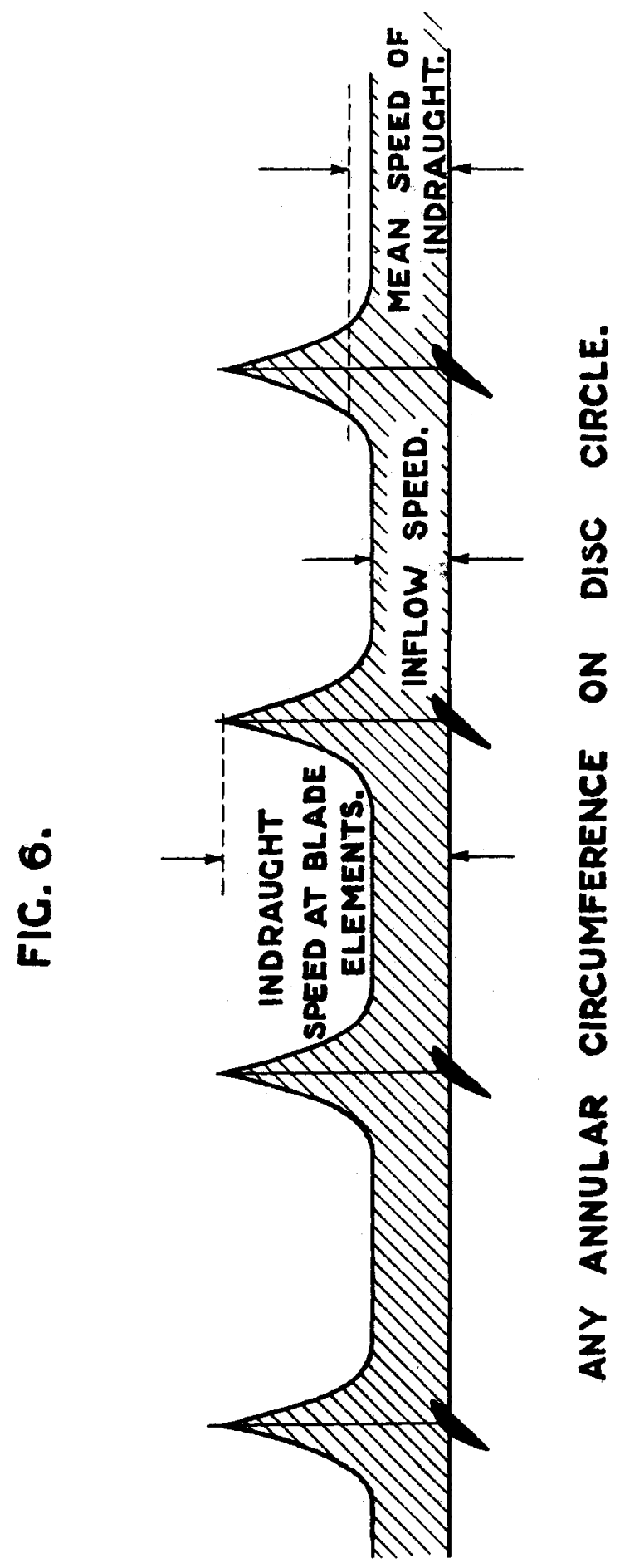


inflow speed at the blade elements for the same radii.* Notice, in passing, that if the angle of incidence $a_{r}$ is equal to

$$
a_{\mathrm{r}}=\left\{a+\frac{1}{2} \delta m \beta(180 / \pi)\right\} /\left\{\mathrm{I}+\frac{1}{2} m \delta(180 / \pi)\right\}
$$

which is quite a possible value, for we have already seen that $\sigma \delta=\pi / 9$ is of the right order of value and we know that the value of $\sigma$ lies between zero and unity, so that the value of $\delta$ lies between infinity and $\pi / 9$, giving :-

$$
a_{\mathbf{r}}=\beta \text {, when } \delta=\text { infinity }
$$

and $a_{\mathrm{r}}=(a+\operatorname{Iom} \beta) /(\mathrm{I}+\mathrm{Iom})$, when $\delta=\pi / 9$.

A usual value for $m$ being $m=.05$, we get :-

$$
a_{\mathrm{r}}=\left(\alpha+\frac{1}{2} \beta\right) / \mathbf{1} .5 \text { or } \alpha_{\mathbf{r}}=\beta
$$

as the two extreme cases. Neither of these extreme cases, and therefore no case intermediate between then, is in any way an impossible one, so that the above solution for $a_{r}$ is quite a reasonable one. But this solution for $a_{r}$ corresponds to $\sigma q=\frac{1}{2}$, i.e., it corresponds to the inflow/slip ratio, having a value of one-half. This value is of the right order and will be familiar to students of the old "inflow theory." Hence, again we notice that the theory given here gives results of the right order in value. We can now find the values of the axial thrust and the torque of the whole propeller. We write $d T$ for the thrust and $d Q x$ for the torque on the blade element. The total thrust of the propeller is then :-

and the power

$$
N T=N \int(\delta T / \delta x) d x,
$$

$$
N H=2 \pi n N / 55^{\circ} \int(\delta Q / \delta x) x d x
$$

in the $\mathrm{lb}$. $\mathrm{ft}$. sec. system of units. So that we now proceed to find $\delta T / \delta x$ and $\delta Q / \delta x$. From Fig. 5 it is evident that

$d T=d R \cos \left(\gamma+a-a_{\mathbf{r}}\right)$, where $d R=$ resultant force on blade element, and $d R=k_{\mathrm{y}} \sec \gamma \rho b d x U^{2}$, by the ordinary aerofoil equation.

Hence, $d T=\cos \left(a-a_{\mathbf{r}}\right)\left[\mathrm{I}-\tan \gamma \tan \left(a-a_{\mathrm{r}}\right)\right] k_{\mathrm{y}} \rho b d x U^{2}$,

i.e., $\delta T / \delta x=\cos \left(\alpha-a_{\mathrm{r}}\right)\left[\mathrm{I}-\tan \gamma \tan \left(\alpha-a_{\mathrm{r}}\right)\right] k_{\mathrm{y}} \rho b U^{2}$.

But from the geometry of Fig. 5 we have :-

$\mathrm{U} \sin \left(a-a_{\mathrm{r}}\right)=\Sigma_{\mathrm{s}} \cos \left(\gamma+a-a_{\mathrm{r}}\right)$

and

and

giving

$$
U \cos \left(a-a_{r}\right)=C-\Sigma_{s} \sin \left(\gamma+a-a_{r}\right)
$$

This gives:-

$$
\therefore \tan \left(\gamma+a-a_{r}\right)=\left\{C-U \cos \left(a-a_{r}\right)\right\} /\left\{U \sin \left(a-a_{r}\right)\right\}
$$

$$
U=C /\left\{\cos \left(a-a_{r}\right)+\sin \left(a-a_{r}\right) \tan \left(\gamma+a-a_{r}\right)\right\}
$$

and

$$
U=C \cos \left(a-a_{\mathrm{r}}\right)\left[\mathrm{I}-\tan \gamma \tan \left(a-a_{\mathrm{r}}\right)\right]
$$

$$
\begin{gathered}
\therefore \delta T / \delta x=\cos ^{3}\left(a-a_{\mathrm{r}}\right)\left[\mathrm{I}-\tan \gamma \tan \left(a-a_{\mathrm{r}}\right)\right]^{3} k_{\mathrm{y}} \rho b C^{2}, \\
\text { i.e., } \delta T / \delta x=C^{2} k_{y} \rho b\left\{\mathrm{I}-\tan \gamma \tan \left(a-a_{\mathrm{r}}\right)\right\}^{3} /\left\{\mathrm{I}+\tan ^{2}\left(a-a_{\mathrm{r}}\right)\right\}^{3 / 2} \\
=C^{2} k_{\mathrm{y}} \rho b\left\{\mathrm{I}-3 \tan \gamma \tan \left(a-a_{\mathrm{r}}\right)\right\} /\left\{\mathrm{I}+3 / 2 \tan ^{2}\left(a-a_{\mathrm{r}}\right)\right\}
\end{gathered}
$$

Approximately, since both $\tan \gamma \tan \left(a-a_{r}\right)$ and $\tan ^{2}\left(a-a_{r}\right)$ are small compared to unity.

Hence

$$
\begin{gathered}
\delta T / \delta x=C^{2} \rho b\left\{k_{\mathrm{y}}-3 k_{\mathrm{x}} \tan \left(a-a_{\mathrm{r}}\right)\right\} /\left\{\mathbf{I}+3 / 2 \tan ^{2}\left(a-a_{r}\right)\right\} \\
=C^{2} \rho b\left\{m\left(a_{\mathrm{r}}-\beta\right)-k_{\mathrm{x}}(\pi / 60)\left(a-a_{\mathrm{r}}\right)\right\} /\left\{\mathbf{I}+(3 / 2)(\pi / \mathrm{I} 8 \mathrm{o})^{2}\left(a-a_{\mathrm{r}}\right)^{2}\right\}
\end{gathered}
$$

* This assumption is not required for the theory generally. It is used only in evaluating $\theta$ in the last part of the Paper. 
to the same degree of approximation as before, and substituting for $k_{y}$ in terms of $a_{r}$ in the approximate equation:-

$$
k_{y}=m\left(a_{x}-\beta\right)
$$

Hence $(\delta T / \delta x)$ is found in terms of the radius $(x)$ and the angle $\left(\alpha_{\mathrm{r}}\right)$, which latter is known already in terms of the angle of the blade and $q$. The values of $\left(a_{\mathbf{r}}-\beta\right)$ and $\left(a-a_{\mathbf{r}}\right)$ are then evidently:-

and

$$
a_{\mathrm{r}}-\beta=(a-\beta) /(\mathrm{I}+\lambda q) \text {; }
$$

$$
a-a_{r}=\{\lambda q(a-\beta)\} /\{\mathrm{I}+\lambda q\}
$$

Then by substitution:-

$$
\begin{gathered}
\delta T / \delta x=C^{2} \rho b \cdot(a-\beta)(\mathrm{I}+\lambda q)\left\{m-(\pi / 60) k_{\mathbf{z}} \lambda q\right\} / \\
\left\{(\mathrm{I}+\lambda q)^{2}+(3 / 2)(\pi / \mathrm{i} 80)^{2} \lambda^{2} q^{2}(\alpha-\beta)^{2}\right\}
\end{gathered}
$$

giving $(\delta T / \delta x)$ in terms of $\lambda q$, etc.

Now

say where

$$
\begin{gathered}
\lambda q=(\mathrm{1} 8 \mathrm{o} / \pi) \sigma \delta m k(N b / x) \\
=(\mathrm{I} 8 \mathrm{o} / \pi) m(N b / x) \sigma \delta k \\
\text { i.e., } \lambda q=(\mathrm{1} 8 \mathrm{o} / \pi) m(N b / x) \theta,
\end{gathered}
$$

$$
\theta=\sigma \delta k \text {. }
$$

So that $\theta$, or $\sigma \delta k$, becomes now the only unknown quantity in the equation. Since also the term $(\pi / 60) k_{x} \lambda q$ is small compared to the term $(m)$ because it is of equal ratio to $(m)$ to the ratio

$$
k_{\mathrm{x}}(\pi / 60)\left(a-a_{\mathrm{r}}\right) / k_{\mathrm{y}}
$$

which is small compared to unity, except for the case when $k_{\mathrm{y}}$ is in the neighbourhood of $\beta$ the "no lift" angle-an ordinary value being of the order of $1 / 15$ we may in many cases treat it only as of the order of a correction and hence assume the value of $k_{x}$ to be constant with the blade radius. This is a simplification in many cases.

Then substituting for $\lambda q$ in $\delta T / \delta x$, we get :-

$$
\begin{gathered}
\delta T / \delta x=C^{2} \rho b m(a-\beta)\{\mathrm{I}+\theta(\mathrm{I} 8 \mathrm{o} / \pi) m(N b / x)\}\left\{\mathrm{I}-3 \theta k_{\mathrm{x}}(N b / x)\right\} / \\
{\left[\{\mathrm{I}+\theta(\mathrm{I} 8 \mathrm{o} / \pi) m(N b / x)\}^{2}+(3 / 2) \theta^{2} m^{2}(a-\beta)^{2}(N b / x)^{2}\right]}
\end{gathered}
$$

This is the formula for the axial thrust of any type of fan. The only unknown quantity is seen to be $\theta$, and later the value of $\theta$ will be determined to a first approximation.

By a similar process we now proceed to find the value of the torque, i.e., we find the expression for $\delta Q / \delta x$. The circumferential component of the force reaction on any blade element is given by :-

$$
\begin{gathered}
d Q^{\circ}=d R \sin \left(\gamma+a-a_{r}\right) \\
=\sin \left(\gamma+a-a_{\mathrm{r}}\right) k_{\mathrm{y}} \sec \gamma \rho b d x U^{2} \\
i . e ., \delta Q / \delta x=\cos \left(a-a_{\mathrm{r}}\right)\left[\tan \gamma+\tan \left(a-a_{\mathrm{r}}\right)\right] k_{\mathrm{y}} \rho b U^{2} \\
=\cos ^{3}\left(a-a_{\mathrm{r}}\right)\left[\tan \gamma+\tan \left(a-a_{\mathrm{r}}\right)\right] k_{\mathrm{y}} \rho b C^{2}\left[\mathrm{I}-\tan \gamma \tan \left(a-a_{\mathrm{r}}\right)\right]^{2} \\
=C^{2} k_{\mathrm{y}} \rho b\left\{\tan \gamma+\tan \left(a-a_{\mathrm{r}}\right)\right\}\left\{\mathrm{I}-\tan \gamma \tan \left(a-a_{\mathrm{r}}\right)\right\}^{2} /\left\{\mathrm{I}+\tan ^{2}\left(a-a_{\mathrm{r}}\right)\right\}^{s / 2} \\
i . e ., \delta Q / \delta x=C^{2} k_{\mathrm{y}} \rho b\left\{\tan \gamma+\tan \left(a-a_{\mathrm{r}}\right)\right\}\left\{\mathrm{I}-2 \tan \gamma \tan \left(a-a_{\mathrm{r}}\right)\right\} / \\
\left\{\mathrm{I}+(3 / 2) \tan ^{2}\left(a-a_{\mathrm{r}}\right)\right\}
\end{gathered}
$$


Approximately as before,

$$
\begin{gathered}
\text { i.e., } \delta Q / \delta x=C^{2} \rho b\left\{k_{\mathrm{x}}+k_{\mathrm{y}} \tan \left(a-a_{\mathrm{r}}\right)\right\}\left\{k_{\mathrm{y}}-2 k_{\mathrm{x}} \tan \left(a-a_{\mathrm{r}}\right)\right\} / \\
k_{\mathrm{y}}\left\{\mathrm{I}+(3 / 2) \tan ^{2}\left(a-a_{\mathrm{r}}\right)\right\} \\
=C^{2} \rho b\left\{k_{\mathrm{x}}+m(\pi / \mathrm{I} 80)\left(a_{\mathrm{r}}-\beta\right)\left(a-a_{\mathrm{r}}\right)\right\}\left\{m\left(a_{\mathrm{r}}-\beta\right)-k_{\mathrm{x}}(\pi / 90)\left(a-a_{\mathrm{r}}\right)\right\} / \\
m\left(a_{\mathrm{r}}-\beta\right)\left\{\mathrm{I}+(3 / 2)(\pi / 180)^{2}\left(a-a_{\mathrm{r}}\right)^{2}\right\}
\end{gathered}
$$

substituting as before. Then

$$
\begin{gathered}
\delta Q / \delta x=C^{2} \rho b\left\{k_{\mathrm{x}}(\mathrm{I}+\lambda q)^{2}+m(\pi / \mathrm{I} 80) \lambda q(\alpha-\beta)^{2}\right\}\left\{m-k_{\mathrm{x}}(\pi / 90) \lambda q\right\} / \\
m\left\{(\mathrm{I}+\lambda q)^{2}+(3 / 2)(\pi / \mathrm{I} 80)^{2} \lambda^{2} q^{2}(\alpha-\beta)^{2}\right\}
\end{gathered}
$$

Hence, substituting for $\lambda q$ as before, we get finally :-

$$
\begin{gathered}
\delta Q / \delta x \\
=C^{2} \rho b\left[k_{\mathrm{x}}\{\mathrm{I}+\theta(\mathrm{I} 8 \mathrm{o} / \pi) m(N b / x)\}^{2}+m^{2}(a-\beta)^{2} \theta(N b / x)\right]\left[\mathrm{I}-2 k_{\mathrm{x}} \theta(N b / x)\right] / \\
{\left[\{1+\theta(\mathrm{I} 8 \mathrm{o} / \pi) m(N b / x)\}^{2}+(3 / 2) m^{2}(a-\beta)^{2} \theta^{2}(N b / x)^{2}\right]}
\end{gathered}
$$

This is the formula from which the power is obtained for any type of fan, the torque being $\int x(\delta Q / \delta x) d x$ for each blade. As for the axial thrust formula, the only unknown quantity is $\theta$.

\section{Experimental Determination of $\theta$.}

It is evident that $\Sigma_{s}=C \sec \gamma \sin \left(a-a_{r}\right)$. And the vertical component, i.e., the component velocity normal to the screw disc, of $\Sigma_{g}$ is $\Sigma_{g} \cos \left(\gamma+a-a_{r}\right)$. Hence, denoting this normal velocity component by $\Sigma_{8}{ }^{\prime}$, we have:-

$$
\begin{gathered}
\Sigma_{\mathrm{s}}{ }^{\prime}=\Sigma_{\mathrm{s}} \cos \left(\gamma+a-a_{\mathrm{r}}\right) \\
=C \tan \left(a-a_{\mathrm{r}}\right)\left\{\mathrm{I}-\tan \gamma \tan \left(a-a_{\mathrm{r}}\right)\right\} /\left\{\mathrm{I}+\tan ^{2}\left(a-a_{\mathrm{r}}\right)\right\} \\
\text { i.e., } \Sigma_{\mathrm{s}}{ }^{\prime}=C(\pi / \mathrm{I} 80) \lambda q(a-\beta)(\mathrm{I}+\lambda q)\left\{m-k_{\mathrm{x}}(\pi / \mathrm{I} 80) \lambda q\right\} / \\
m\left\{(\mathrm{I}+\lambda q)^{2}+(\pi / \mathrm{I} 80)^{2} \lambda^{2} q^{2}(a-\beta)^{2}\right\}
\end{gathered}
$$

in terms of $\lambda q$ to the same order of approximation. And

$$
\begin{aligned}
\therefore \Sigma_{\mathrm{s}}{ }^{\prime}= & m C \theta(N b / x)(a-\beta)\{\mathrm{I}+(\mathrm{I} 80 / \pi) m \theta(N b / x)\}\left\{\mathrm{I}-k_{\mathrm{x}} \theta(\mathrm{Nb} / \mathrm{x})\right\} / \\
& {\left[(\mathrm{I}+(\mathrm{I} 80 / \pi) m \theta(N b / x)\}^{2}+(a-\beta)^{2} m^{2} \theta^{2}(N b / x)^{2}\right] }
\end{aligned}
$$

in terms of the radius $x$ and the unknown quantity $\theta$.

Now compare this result with the formula for $\delta T / \delta x$; and notice that, since the terms in the denominators involving $(\alpha-\beta)^{2}$ are very small in practically every case, we can neglect them in both formulæ for a first approximation in finding a tentative value for $\theta$. Then approximately :-

$$
\Sigma_{\mathrm{s}}^{\prime}=m C \theta(N b / x)(\alpha-\beta)\left\{\mathrm{I}-k_{\mathrm{x}} \theta(N b / x)\right\} /\{\mathrm{I}+(\mathrm{I} 8 \mathrm{o} / \pi) m \theta(N b / x)\}
$$

And similarly for $\delta T / \delta x$ approximately :-

$$
\delta T / \delta x=C^{2} \rho b m(a-\beta)\left\{\mathrm{I}-\mathfrak{3}^{\theta} \theta k_{\mathrm{x}}(N b / x)\right\} /\{\mathrm{I}+\theta(\mathrm{r} 8 \mathrm{o} / \pi) m(N b / x)\}
$$

Now since

$$
\begin{gathered}
m C(a-\beta) /\{\mathbf{I}+\theta(\mathbf{I} 80 / \pi) m(N b / x)\} \\
=\Sigma_{s}^{\prime} / \theta(N b / x)\left\{\mathbf{I}-k_{\mathbf{x}} \theta(N b / x)\right\} \\
\therefore \delta T / \delta x=C \rho b\left\{\mathbf{I}-3 \theta k_{\mathbf{x}}(N b / x)\right\} \Sigma_{\mathbf{s}} /\left\{\theta(N b / x)\left[\mathbf{I}-k_{\mathbf{x}} \theta(N b / x)\right]\right\}
\end{gathered}
$$

in terms of $\Sigma_{\mathrm{s}}^{\prime}$.

Hence, if we have any experimental fan which has a constant value of $\Sigma_{s}{ }^{\prime}$ for all radii $x$ we can employ the above formula to find the thrust of the fan, and conversely, if we know the thrust of the fan and the value of $\Sigma_{s}^{\prime}$ we can determine the value of $\theta$. Further, it is evident that for all "good" angles of incidence $a_{r}, \tan \gamma$ is small compared to unity and $\tan \gamma \tan \left(a-a_{r}\right)$ is very small 


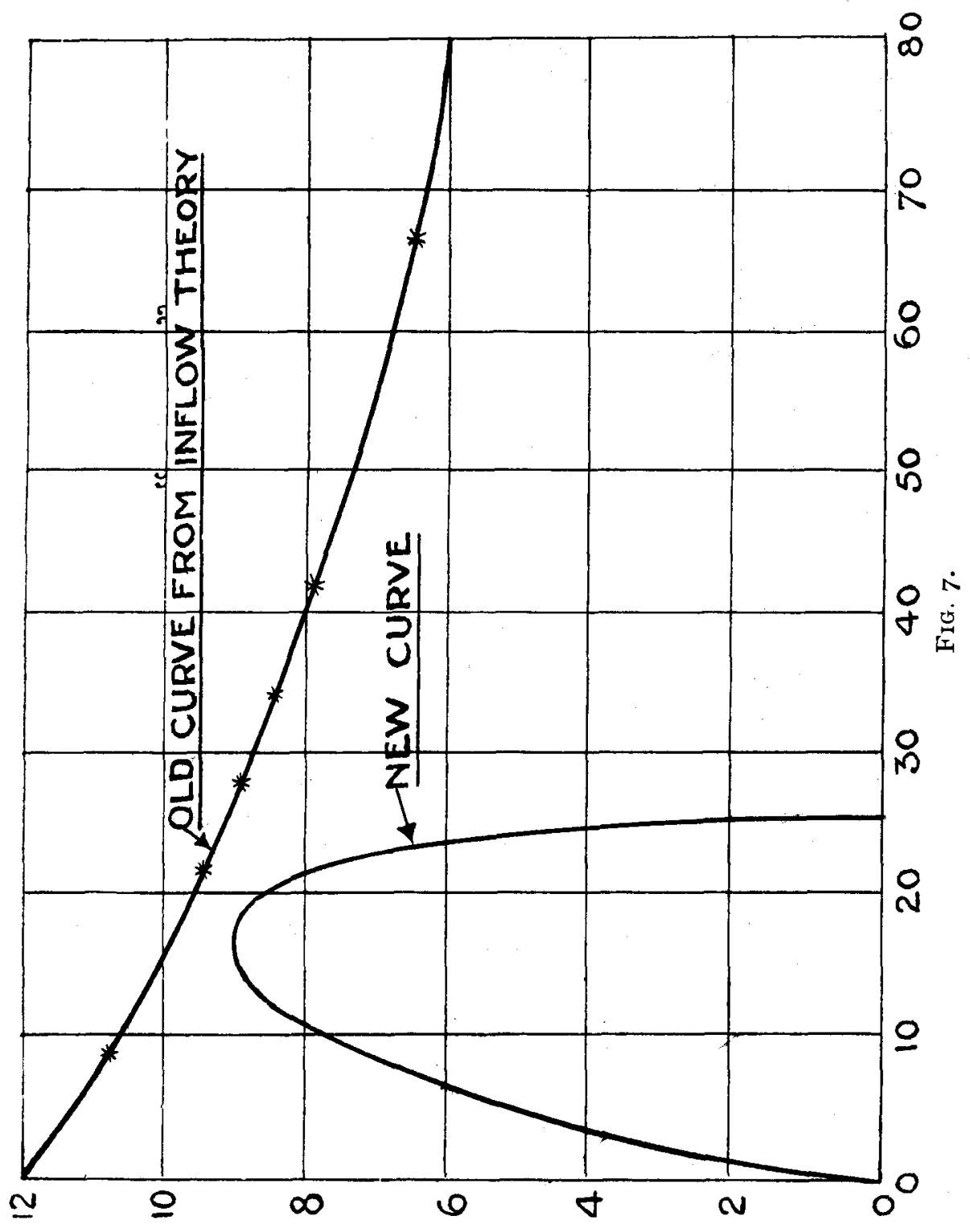


except quite near the boss where the thrust is vanishingly small, so that a further approximation gives :-

and $\therefore$

$$
\delta T / \delta x=C \rho b \Sigma_{\mathrm{s}}{ }^{\prime} / \theta(\mathrm{Nb} / x)
$$

$$
\theta=\rho \Sigma_{s}^{\prime} / T \int b C d x /(N b / x)
$$

from which the value of $\theta$ is at once found. Some years ago I made and tested two wooden fans, both of which were geometrically identical and of equal diameter. The diameter was three feet. One was a two-bladed and the other a four-bladed fan. They both gave identical results on test as near as I was able to measure at the time. In each fan the product (chord blade width $x$ number of blades) was the same and equal to $4 / 3$ feet, so that the four-bladed fan had blades one-half the chord width of the other. The chord blade width was constant along the blade length. The r.p.m. was in each case $1,05^{\circ}$, the power being supplied by an electric motor of 0.6 b.h.p. rating running at 1,400 r.p.m., the reduction to the fan being by a flat belt drive of $3: 4$ reduction. The axial thrust recorded was a trifle over $12 \mathrm{lbs}$, and the speed of in-draught normal to the screw disc was found by. an anemometer to be approximately constant with blade radius and equal to about 16 feet per second.* The speed of outdraught was measured as about double this figure. From these admittedly rather rough test results I propose now to calculate the value of $\theta$ as a first tentative estimate.

$$
\begin{gathered}
\theta=\rho \Sigma_{s}{ }^{\prime} / T \int b C d x /(N b / x) \\
T=\mathrm{I} 2 \mathrm{lbs} \cdot N=\text { thrust per blade. } \\
=\left(.00237 \times \mathrm{I} 6 \times 2 \pi \times \mathrm{I} 7 \frac{1}{2}\right) /(\mathrm{I} 2) \int_{a}^{3 / 2} x^{2} d x \\
=\left(.00237 \times 16 \times 2 \pi \times 17 \frac{1}{2} \times 27\right) /(\mathrm{I} 2 \times 3 \times 8)=.39 \mathrm{I} .
\end{gathered}
$$

Hence, $\theta=.39 \mathrm{I}$ as a first rough estimate.

\section{Conclusions.}

The theory enunciated above has been developed in order to, if possible, take the place of the "inflow" theory of the airscrew, which latter is too empirical, although the present paper is concerned only with the special case of the propeller working at a fixed point, without axial motion, after the manner of a fan. The theory given here is based upon blade interference and nothing else, and is amalgamated with and forms a correction upon the original "blade element" theory of Drzewiecki. It requires further development so as to include the general case of propeller action, but it is thought that the present paper will have achieved its main object if it draws attention to the fact that the "inflow" theory being admitted to be of too highly empirical a nature, a theory can be evolved which shall possess the same power in application as this older theory and which, at the same time, shall rest upon a more rational basis. The key and corner stone of the new theory is blade interference, the regime of which has only recently begun to be understood in its relation to all screw propeller action. Not only does this new theory not require the assumption-upon which the "inflow" theory depends-that the inflow/slip ratio is a constant, but it shows that this ratio varies and how it varies, the form of which variation is confirmed by experiment.

* Or slightly greater. It is here assumed that this figure is approximately equal to the normal inflow speed component. 


\section{APPENDIX.}

From the two equations, when $\Sigma_{s}^{\prime}$ is a constant with radius:-

and

$$
\delta T / \delta x=\left(C_{\rho} b \Sigma_{\mathrm{s}}\right) / \theta(N b / x),
$$

$$
\delta Q / \delta x=\rho b\left[C^{2} k_{\mathrm{x}}+\left(\Sigma_{\mathrm{s}}{ }^{\prime}\right)^{2} / \theta(\mathrm{Nb} / x)\right],
$$

we can determine the thrust $T$ in terms of the power $H$ and propeller diameter $D$. Assume constant blade width $b$. Then :-

$$
N T=2 \pi n \rho \Sigma_{\mathrm{s}}^{\prime} / \theta \int_{0}^{D / 2} x^{2} d x=\left(2 \pi n \rho \Sigma_{s}^{\prime} / 3^{\theta}\right)\left(D^{3} / 8\right)
$$

$N T=\left(\pi n \rho \Sigma_{3}{ }^{\prime} D^{3}\right) /(\mathrm{I} 2 \theta)=$ total axial thrust of propeller.

And

$$
\begin{aligned}
& N(\delta Q / \delta x) x=\rho N b C^{2} k_{\mathrm{x}} x+\left(\rho \Sigma_{\mathrm{s}}{ }^{2} x^{2}\right) / \theta \\
& N H=2 \pi n N / 55^{\circ} \int(\delta Q / \delta x) x d x=2 \pi n \rho / 55^{\circ} \int\left[N b C^{2} x k_{\mathrm{x}}+\left(\Sigma_{\mathrm{g}}{ }^{2} x^{2}\right) / \theta\right] d x \\
& =2 \pi n \rho / 55^{\circ}\left[N b k_{\mathrm{x}} 4 \pi^{2} n^{2} \int_{1+0}^{D / 2} x^{3} d x+\left(\Sigma_{\mathrm{s}}{ }^{2} / \theta\right) \int_{0}^{D / 2} x^{2} d x\right] \\
& =2 \pi n \rho / 55^{\circ}\left[\left(N b k_{x^{2}} \pi^{2} n^{2}\right)\left(D^{4} / \mathrm{L} 6\right)+\left(\Sigma_{\mathrm{s}}{ }^{2} / 3^{\theta} \theta\right)\left(D^{3} / 8\right)\right] \\
& =2 \pi n \rho / 55^{\circ}\left[\left(N b k_{x} \pi^{2} n^{2} D^{4} / \mathrm{l} 6\right)+6 \theta(N T)^{2} / \pi^{2} n^{2} \rho^{2} D^{3}\right]
\end{aligned}
$$

and

and

$$
\therefore 6 \theta(N T)^{2} / \pi^{2} n^{2} \rho^{2} D^{3}=55^{\circ}(N H) / 2 \pi n \rho-N b k_{\mathrm{x}} \pi^{2} n^{2} D^{4} / 16
$$

$$
\therefore(N T)^{2}=\left\{\left(55^{\circ}(N H) / 2 \pi n \rho\right)-\left(N b k_{x} \pi^{2} n^{2} D^{4} / 16\right)\right\}\left(\pi^{2} n^{2} \rho^{2} D^{3} / 6 \theta\right)
$$

This gives the axial thrust of any fan, of constant chord blade width and constant normal component of inflow speed, in terms of blade width.

Suppose we put $N b=\phi D$, so that the total blade width becomes a fraction $(\phi)$ of the propeller diameter $D$. Then we get, writing $W$ for the total axial thrust $(N T)$ and $H$ for the total $B H P$ instead of $N H$.

$$
\left.W=\left\{\pi^{\frac{1}{2}} n^{\frac{1}{2}} \rho^{\frac{1}{2}} D^{3 / 2} \sqrt{55^{\circ}} H^{\frac{1}{2}} / \sqrt{12 \theta}\right\} \sqrt{\left\{\mathrm{I}-\left(\phi k_{\mathrm{x}} \pi^{3} n^{3} D^{5} \rho\right) / 8.55^{\circ} H\right.}\right\}
$$

Now I have previously shown elsewhere* that the thrust of any fan.or helicopter can be represented by :-

$$
W=\rho^{\frac{1}{3}} H^{\frac{2}{3}} D^{\frac{2}{3}} f f\left(n D^{5 / 3} \rho^{\frac{1}{3}} / H^{\frac{1}{3}}\right)
$$

So that by equating these two equations we can find the form of the unknown function $f$. This gives :-

$$
f\left(n D^{5 / 3} \rho^{\mathbf{t}} / H^{t}\right)=\left(55^{\circ} \pi / \mathrm{I} 2 \theta\right)^{\frac{1}{2}}\left(n D^{5 / 3} \rho^{\frac{2}{2}} / H^{t}\right)^{\frac{1}{2}}\left\{\mathrm{I}-\left(\phi k_{\mathrm{x}} \pi^{3} / 4 \cdot 400\right)\left(n D^{5 / 3} \rho^{\frac{1}{8}} / H^{t}\right)^{3}\right\}^{\frac{1}{3}}
$$

So that, writing $\psi$ for the argument $\left(n D^{5 / 3} \rho^{\frac{1}{3}} / H^{3}\right)$, we get :-

$$
f(\psi)=\left(55^{\circ} \pi / \text { 1 } 2 \theta\right)^{\frac{1}{2}} \psi^{\frac{2}{2}}\left\{1-\phi k_{x} \pi^{3} \psi^{3} / 4.400\right\}^{\frac{1}{2}}
$$

And the form of the function $f(\psi)$ is hence determinate and both $\psi$ and $f(\psi)$ are non-dimensional.

Now I have shown elsewhere that if we take

so that

$$
F(\psi)=\rho^{t} f(\psi)
$$

$$
W=H^{2} D F(\psi)
$$

the value of $F(\psi)$ is round about 8 for many cases. 
Now in this new theory we find that the corresponding value of $F(\psi)$ is given by :-

$$
F(\psi)=\rho^{\frac{1}{3}}\left(55^{\circ} \pi / \mathrm{I}_{2} \theta\right)^{\frac{1}{2}} \psi^{\frac{1}{2}}\left\{\mathrm{I}-\phi k_{\mathrm{x}} \pi^{3} \psi^{3} / 4400\right\}^{\frac{1}{2}}
$$

where the value of $\rho$ is the "ground level" value of .00237 , and $\rho=1 / 7 \cdot 5=2 / 15$, so that

$$
F(\psi)=(2 / 15)(550 \pi / \mathrm{I} 2 \times 39 \mathrm{I}) \psi^{\frac{1}{2}}\left\{\mathrm{I}-\phi k_{\mathbf{x}} \pi^{3} \psi^{3} / 4400\right\}^{\frac{1}{2}}
$$

with $\theta=.39$ as found already. Then

$$
F(\psi)=2.5^{6} \psi^{\frac{1}{2}}\left\{\mathrm{I}-\phi k_{\mathrm{x}} \pi^{3} \psi^{3} / 4400\right\}^{\frac{1}{2}}
$$

Now in order to get an idea of the order of values let us take some rough values for $\phi k_{\mathrm{x}}$. Let $k_{\mathrm{x}}=\mathrm{I} / 60, \phi=\frac{1}{2}$ (not too large for a fan or helicopter), so that

Hence,

$$
\phi k_{\mathrm{x}} \pi^{3} / 4400=3^{I} /(2 \times 60 \times 4400)=1 / 17,000
$$

$$
F(\psi)=2.56 \psi^{\frac{1}{2}}\left(\mathrm{r}-\psi^{3} / 17,000\right)^{\frac{1}{2}}
$$

showing that for small values of $\psi$ the last term in the equation is only a small correction, but for values of $\psi$ of 20 and over the correction is very material.

We can now form the following characteristic table of values :-

$\begin{array}{ccccccc}\psi & 0 & 5 & 10 & \text { I } 5 & 20 & 25.7 \\ F^{\prime}(\psi) & 0 & 5 \cdot 7 & 7.83 & 8.87 & 8.35 & 0\end{array}$

This is plotted in Fig. 7, together with the curve obtained from the " inflow", theory and published by me in "The Helicopter Flying Machine" (Aircraft Engineering, June, I920, Fig. 5). It will be seen that although the two curves are so dissimilar in form the values of $F(\psi)$ are of the same order in the two curves in the " useful" region from $\psi=10$ to $\psi=20$, and that in this region the value of $F(\psi)$ is round about 8 , as mentioned in the article referred to above. Thus, the new theory of blade interference is seen to give results of the right order in this case also.

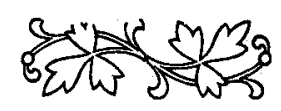

